\title{
On ergodicity of some cylinder flows
}

\author{
by
}

Krzysztof Frączek (Toruń)

Abstract. We study ergodicity of cylinder flows of the form

$$
T_{f}: \mathbb{T} \times \mathbb{R} \rightarrow \mathbb{T} \times \mathbb{R}, \quad T_{f}(x, y)=(x+\alpha, y+f(x)),
$$

where $f: \mathbb{T} \rightarrow \mathbb{R}$ is a measurable cocycle with zero integral. We show a new class of smooth ergodic cocycles. Let $k$ be a natural number and let $f$ be a function such that $D^{k} f$ is piecewise absolutely continuous (but not continuous) with zero sum of jumps. We show that if the points of discontinuity of $D^{k} f$ have some good properties, then $T_{f}$ is ergodic. Moreover, there exists $\varepsilon_{f}>0$ such that if $v: \mathbb{T} \rightarrow \mathbb{R}$ is a function with zero integral such that $D^{k} v$ is of bounded variation with $\operatorname{Var}\left(D^{k} v\right)<\varepsilon_{f}$, then $T_{f+v}$ is ergodic.

1. Introduction. Assume that $T:(X, \mathcal{B}, \mu) \rightarrow(X, \mathcal{B}, \mu)$ is an ergodic measure-preserving automorphism of a standard Borel space. Each measurable function $f: X \rightarrow \mathbb{R}$ is called a cocycle. For every $n \in \mathbb{Z}$, let

$$
f^{(n)}(x)= \begin{cases}f(x)+f(T x)+\ldots+f\left(T^{n-1} x\right) & \text { if } n>0, \\ 0 & \text { if } n=0, \\ -\left(f\left(T^{n} x\right)+f\left(T^{n+1} x\right)+\ldots+f\left(T^{-1} x\right)\right) & \text { if } n<0 .\end{cases}
$$

Let $\overline{\mathbb{R}}=\mathbb{R} \cup\{\infty\}$ be the one-point Aleksandrov compactification of $\mathbb{R}$. Then $r \in \overline{\mathbb{R}}$ is said to be an extended essential value of $f$ (see [10]) if for each open neighbourhood $U(r)$ of $r$ and an arbitrary set $C \in \mathcal{B}$ with $\mu(C)>0$, there exists an integer $n$ such that

$$
\mu\left(C \cap T^{-n} C \cap\left\{x \in X: f^{(n)} \in U(r)\right\}\right)>0 .
$$

The set of extended essential values will be denoted by $\bar{E}(f)$. The set $E(f)=$ $\bar{E}(f) \cap \mathbb{R}$ is called the set of essential values of $f$. The skew product

$$
T_{f}:(X \times \mathbb{R}, \widetilde{\mathcal{B}}, \widetilde{\mu}) \rightarrow(X \times \mathbb{R}, \widetilde{\mathcal{B}}, \widetilde{\mu}), \quad T_{f}(x, y)=(T x, y+f(x)),
$$

2000 Mathematics Subject Classification: Primary 37A05, 37C40.

Research partly supported by KBN grant 2 P301 03107 (1994) and by Foundation for Polish Science. 
is said to be the cylinder flow. Here $\widetilde{\mu}$ denotes the product measure of $\mu$ and infinite Lebesgue measure on the line. It is shown in [10] that $E(f)$ is a closed subgroup of $\mathbb{R}$ and it is the collection of periods of $T_{f}$-invariant functions, i.e.

$$
E(f)=\left\{r \in \mathbb{R}: \forall_{\phi: X \times \mathbb{R} \rightarrow \mathbb{R}, \phi \circ T_{f}=\phi} \phi(x, y+r)=\phi(x, y) \widetilde{\mu} \text {-a.e. }\right\} .
$$

In particular, $T_{f}$ is ergodic iff $E(f)=\mathbb{R}$. if

We say that a strictly increasing sequence $\left\{q_{n}\right\}_{n \in \mathbb{N}}$ is a rigid time for $T$

$$
\lim _{n \rightarrow \infty} \mu\left(T^{q_{n}} A \triangle A\right)=0 \quad \text { for any } A \in \mathcal{B} .
$$

In [6], Lemańczyk, Parreau and Volný have proved

Proposition 1. Suppose that $f: X \rightarrow \mathbb{R}$ is an integrable cocycle such that the sequence $\left\{\left\|f^{\left(q_{n}\right)}\right\|_{L^{1}}\right\}_{n \in \mathbb{N}}$ is bounded, where $\left\{q_{n}\right\}_{n \in \mathbb{N}}$ is a rigid time for $T$. If

$$
\limsup _{n \rightarrow \infty}\left|\int_{X} e^{2 \pi i l f^{\left(q_{n}\right)}} d \mu\right| \leq c<1
$$

for all $l$ large enough, then $T_{f}$ is ergodic.

We denote by $\mathbb{T}$ the group $\mathbb{R} / \mathbb{Z}$ which will be identified with the interval $[0,1)$ with addition mod 1 . Let $\lambda$ denote the Lebesgue measure on $\mathbb{T}$. Let $\widetilde{<} \subset \mathbb{T} \times \mathbb{T}$ be defined by: $x \widetilde{<} y$ iff $0<y-x<1 / 2$, where $<\subset \mathbb{T} \times \mathbb{T}$ is the usual order on $[0,1)$. By $\{t\}$ we denote the fractional part of $t$ and $\|t\|$ is the distance of $t$ from the set of integers.

Assume that $\alpha \in[0,1)$ is an irrational with continued fraction expansion

$$
\alpha=\left[0 ; a_{1}, a_{2}, \ldots\right] \text {. }
$$

The natural numbers $a_{n}$ are said to be the partial quotients of $\alpha$. Put

$$
\begin{array}{lll}
r_{0}=0, & r_{1}=1, & r_{n+1}=a_{n+1} r_{n}+r_{n-1}, \\
s_{0}=1, & s_{1}=a_{1}, & s_{n+1}=a_{n+1} s_{n}+s_{n-1} .
\end{array}
$$

The rationals $r_{n} / s_{n}$ are called the convergents, and $s_{n}$ is the $n$th denominator of $\alpha$. We have the inequality

$$
\frac{1}{2 s_{n} s_{n+1}}<\left|\alpha-\frac{r_{n}}{s_{n}}\right|<\frac{1}{s_{n} s_{n+1}} .
$$

For every nonnegative integer $k$, let $S_{k}$ denote the subset of irrational numbers $\alpha$ such that

$$
\liminf _{n \rightarrow \infty} s_{n}^{k+1}\left\|s_{n} \alpha\right\|<\infty
$$

and let $S_{k}^{0}$ denote the subset of irrational numbers $\alpha$ such that

$$
\liminf _{n \rightarrow \infty} s_{n}^{k+1}\left\|s_{n} \alpha\right\|=0 .
$$

The above sets are residual in $\mathbb{T}$. 
A function $f: \mathbb{T} \rightarrow \mathbb{R}$ is said to be piecewise absolutely continuous (PAC for short) if there are $\beta_{0}, \ldots, \beta_{k} \in \mathbb{T}$ such that $\left.f\right|_{\left(\beta_{j}, \beta_{j+1}\right)}$ is absolutely continuous $\left(\beta_{k+1}=\beta_{0}\right)$. Set

$$
f_{+}(x)=\lim _{y \rightarrow x^{+}} f(y) \quad \text { and } \quad f_{-}(x)=\lim _{y \rightarrow x^{-}} f(y) .
$$

Let $a_{j}=f_{+}\left(\beta_{j}\right)-f_{-}\left(\beta_{j}\right)$ for $j=0, \ldots, k$ and

$$
S(f)=\sum_{j=0}^{k} a_{j}=-\sum_{j=0}^{k} f_{-}\left(\beta_{j}\right)-f_{+}\left(\beta_{j}\right)=-\int_{\mathbb{T}} D f(x) d \lambda(x) .
$$

Assume that $\alpha \in[0,1)$ is irrational. Denote by $T x=x+\alpha \bmod 1$ the corresponding ergodic rotation on $\mathbb{T}$. We shall study skew products of the form

$$
T_{f}: \mathbb{T} \times \mathbb{R} \rightarrow \mathbb{T} \times \mathbb{R}, \quad T_{f}(x, y)=(T x, y+f(x)),
$$

where $f: \mathbb{T} \rightarrow \mathbb{R}$ is a measurable cocycle with $\int_{\mathbb{T}} f d \lambda=0$.

In [8], Pask has given a class of cocycles which are PAC with $S(f) \neq 0$, and has showed ergodicity for all irrationals $\alpha$. Lemańczyk, Parreau and Volný [6] have proved that the class of cocycles considered in [8] is ergodically stable in the space $\mathrm{BV}(\mathbb{T})_{0}$ of bounded variation functions with zero integral, i.e. if $f \in \mathrm{PAC}$ with $S(f) \neq 0$ and $\operatorname{Var}(f-g)<|S(f)|$, then $T_{g}$ is still ergodic. It has been proved in [9] that if $f$ is $k-1$ times differentiable a.e. and $D^{k-1} f$ is PAC with $S\left(D^{k-1} f\right) \neq 0$, then $T_{f}$ is ergodic for $\alpha \in S_{k}$.

The aim of this paper is to study the ergodicity of $T_{f}$ in the case where a derivative $D^{k} f$ of $f$ is piecewise absolutely continuous (but not continuous) and $S\left(D^{k} f\right)=0$.

Let $k$ be a natural number. We denote by $C_{0}^{k+\mathrm{BV}}$ the space of $k-1$ differentiable functions $f: \mathbb{T} \rightarrow \mathbb{R}$ with zero integral such that $D^{k-1} f$ is absolutely continuous and $D^{k} f$ is of bounded variation. Set $C_{0}^{0+\mathrm{BV}}=\mathrm{BV}_{0}$.

Observe that if $f: \mathbb{T} \rightarrow \mathbb{R}$ is a function of bounded variation with zero integral, then

$$
\sup _{x \in \mathbb{T}}|f(x)| \leq \operatorname{Var}(f) .
$$

Notice that if $f \in C_{0}^{k+\mathrm{BV}}$, then $\operatorname{Var}\left(D^{j-1} f\right) \leq \operatorname{Var}\left(D^{j} f\right)$ for $j=1, \ldots, k$. Indeed, since $D^{j-1} f$ is absolutely continuous, we have $\operatorname{Var}\left(D^{j-1} f\right)=$ $\int_{\mathbb{T}}\left|D^{j} f\right| d \lambda$ and $\int_{\mathbb{T}} D^{j} f d \lambda=0$. From (1) we have

$$
\operatorname{Var}\left(D^{j-1} f\right)=\int_{\mathbb{T}}\left|D^{j} f\right| d \lambda \leq \sup _{x \in \mathbb{T}}\left|D^{j} f(x)\right| \leq \operatorname{Var}\left(D^{j} f\right) .
$$

In $C_{0}^{k+\mathrm{BV}}$ we define the norm $\|f\|_{k+\mathrm{BV}}=\operatorname{Var}\left(D^{k} f\right)$. With this norm, $C_{0}^{k+\mathrm{BV}}$ becomes a Banach space. Let $C_{0}^{k+\mathrm{PAC}}$ denote the subspace of functions $f \in C_{0}^{k+\mathrm{BV}}$ such that $D^{k} f$ is piecewise absolutely continuous and let $C_{0}^{k+A C}$ 
denote the space of functions $f \in C_{0}^{k+P A C}$ such that $D^{k} f$ is absolutely continuous. Recall that the subspace of trigonometric polynomials is dense in $C_{0}^{k+\mathrm{AC}}$ with respect to the $C_{0}^{k+\mathrm{BV}}$ norm.

Assume that $f \in C_{0}^{k+\mathrm{PAC}}$ and $S\left(D^{k} f\right)=0$. Suppose that $\alpha \in S_{k}^{0}$ and $0=\beta_{0}<\beta_{1}<\ldots<\beta_{d}<1$ are all the discontinuity points of $D^{k} f$. In this paper we will prove the following theorem.

TheOREM 1.1 (Main Theorem). Let $k \in \mathbb{N}$ and $f \in C_{0}^{k+\mathrm{PAC}}$ be such that $S\left(D^{k} f\right)=0$. If there exists a sequence $\left\{q_{n}\right\}_{n \in \mathbb{N}}$ of denominators of $\alpha$ such that

$$
\lim _{n \rightarrow \infty} q_{n}^{k+1}\left\|q_{n} \alpha\right\|=0 \quad \text { and } \quad \lim _{n \rightarrow \infty}\left\{q_{n} \beta_{i}\right\}=\gamma_{i},
$$

where $\gamma_{i} \neq \gamma_{j}$ for $i \neq j, i, j=0, \ldots, d$, then $T_{f}$ is ergodic. Moreover, there exists $\varepsilon>0$ such that if $v \in C_{0}^{k+\mathrm{BV}}$ and $\|v\|_{k+\mathrm{BV}}<\varepsilon$, then $T_{f+v}$ is ergodic.

2. Some generalizations of the Denjoy-Koksma inequality. In this section we prove some generalizations of the Denjoy-Koksma inequality which will be needed to prove the main theorem. Let $Q_{n}$ be a partition of $\mathbb{T}$ into the intervals defined by the points $\{i \alpha\}_{i=0}^{s_{n}-1}$. Then for all $n$, each interval of $Q_{n}$ has length $\left\|s_{n-1} \alpha\right\|+\left\|s_{n} \alpha\right\|$ or $\left\|s_{n-1} \alpha\right\|$.

TheOREM 2.1. For a given nonnegative integer $k$ there is a positive constant $M_{k}=M$ such that if $f \in C_{0}^{k+\mathrm{BV}}$, then

$$
s_{n}^{k}\left|f^{\left(s_{n}\right)}(x)\right| \leq M\left(1+s_{n}^{k+1}\left\|s_{n} \alpha\right\|\right) \operatorname{Var}\left(D^{k} f\right)
$$

for any natural $n$.

Pr o of (by induction on $k$ ). For $k=0$ the inequality (2) is the ordinary Denjoy-Koksma inequality (see [5], p. 73).

Assuming (2) to hold for a certain $k$, we will prove that there exists $M_{k+1}>0$ such that if $f \in C_{0}^{k+1+\mathrm{BV}}$, then

$$
s_{n}^{k+1}\left|f^{\left(s_{n}\right)}(x)\right| \leq M_{k+1}\left(1+s_{n}^{k+2}\left\|s_{n} \alpha\right\|\right) \operatorname{Var}\left(D^{k+1} f\right) .
$$

Let $I$ be an interval of size $\left\|s_{n-1} \alpha\right\|$. Then

$$
\left|\int_{I} f^{\left(s_{n}\right)}(x) d x\right|=\left|\int_{\bigcup_{i=0}^{s_{n}-1} T^{i} I} f(x) d x\right|=\left|\int_{\mathbb{T} \backslash \bigcup_{i=0}^{s_{n}-1} T^{i} I} f(x) d x\right| .
$$

Since

$$
\mathbb{T} \backslash \bigcup_{i=0}^{s_{n}-1} T^{i} I=\bigcup_{j=0}^{s_{n-1}-1} T^{j} J
$$

where $J$ is an interval of size $\left\|s_{n} \alpha\right\|$, we have 


$$
\left|\int_{I} f^{\left(s_{n}\right)}(x) d x\right|=\left|\int_{J} f^{\left(s_{n-1}\right)}(x) d x\right| \leq|J| \operatorname{Var}(f) \leq\left\|s_{n} \alpha\right\| \operatorname{Var}\left(D^{k+1} f\right) .
$$

If $|I|=\left\|s_{n-1} \alpha\right\|+\left\|s_{n} \alpha\right\|$, then we split this into two chunks, one $I_{1}$ of size $\left\|s_{n-1} \alpha\right\|$, the other $I_{2}$ of size $\left\|s_{n} \alpha\right\|$. Then

$$
\left|\int_{I} f^{\left(s_{n}\right)}(x) d x\right|=\left|\int_{I_{1}} f^{\left(s_{n}\right)}(x) d x\right|+\left|\int_{I_{2}} f^{\left(s_{n}\right)}(x) d x\right| \leq 2\left\|s_{n} \alpha\right\| \operatorname{Var}\left(D^{k+1} f\right) .
$$

It follows that for each interval $I$ of $Q_{n}$ there is $x_{I} \in I$ with

$$
\left|f^{\left(s_{n}\right)}\left(x_{I}\right)\right| \leq 4 s_{n}\left\|s_{n} \alpha\right\| \operatorname{Var}\left(D^{k+1} f\right) .
$$

Indeed, if $\left.f^{\left(s_{n}\right)}\right|_{I}$ changes sign, then we can take $x_{I}$ such that $f^{\left(s_{n}\right)}\left(x_{I}\right)=0$. Assume that $\left.f^{\left(s_{n}\right)}\right|_{I}$ does not change sign. Suppose that

$$
\left|f^{\left(s_{n}\right)}(x)\right| \geq 4 s_{n}\left\|s_{n} \alpha\right\| \operatorname{Var}\left(D^{k+1} f\right)
$$

for any $x \in I$. Then

$$
\left|\int_{I} f^{\left(s_{n}\right)}(x) d x\right|>|I| 4 s_{n}\left\|s_{n} \alpha\right\| \operatorname{Var}\left(D^{k+1} f\right)>2\left\|s_{n} \alpha\right\| \operatorname{Var}\left(D^{k+1} f\right),
$$

a contradiction. Since $f$ is absolutely continuous and the formula (2) is true for $k$, we have

$$
\begin{aligned}
\left|f^{\left(s_{n}\right)}(b)-f^{\left(s_{n}\right)}(a)\right| & =\left|\int_{a}^{b} D f^{\left(s_{n}\right)}(x) d x\right| \\
& \leq M_{k}\left(1+s_{n}^{k+1}\left\|s_{n} \alpha\right\|\right) \operatorname{Var}\left(D^{k+1} f\right) \frac{|b-a|}{s_{n}^{k}}
\end{aligned}
$$

for all $a, b \in \mathbb{T}$. If $x \in I \in Q_{n}$, then

$$
\begin{aligned}
\left|f^{\left(s_{n}\right)}(x)-f^{\left(s_{n}\right)}\left(x_{I}\right)\right| & \leq 2 \frac{\left\|s_{n-1} \alpha\right\|}{s_{n}^{k}} M_{k}\left(1+s_{n}^{k+1}\left\|s_{n} \alpha\right\|\right) \operatorname{Var}\left(D^{k+1} f\right) \\
& \leq \frac{2 M_{k}}{s_{n}^{k+1}}\left(1+s_{n}^{k+1}\left\|s_{n} \alpha\right\|\right) \operatorname{Var}\left(D^{k+1} f\right)
\end{aligned}
$$

and finally

$$
\begin{aligned}
s_{n}^{k+1}\left|f^{\left(s_{n}\right)}(x)\right| & \leq s_{n}^{k+1}\left|f^{\left(s_{n}\right)}(x)-f^{\left(s_{n}\right)}\left(x_{I}\right)\right|+s_{n}^{k+1}\left|f^{\left(s_{n}\right)}\left(x_{I}\right)\right| \\
& \leq\left(2 M_{k}\left(1+s_{n}^{k+1}\left\|s_{n} \alpha\right\|\right)+4 s_{n}^{k+2}\left\|s_{n} \alpha\right\|\right) \operatorname{Var}\left(D^{k+1} f\right) \\
& \leq\left(2 M_{k}+4\right)\left(1+s_{n}^{k+2}\left\|s_{n} \alpha\right\|\right) \operatorname{Var}\left(D^{k+1} f\right) .
\end{aligned}
$$

Corollary 2.1. Assume that $\alpha \in S_{k}$ and $\left\{q_{n}\right\}_{n \in \mathbb{N}}$ is a sequence of denominators of $\alpha$ such that the sequence $\left\{q_{n}^{k+1}\left\|q_{n} \alpha\right\|\right\}_{n \in \mathbb{N}}$ is bounded. Then there is a constant $K \geq 1$ such that

$$
q_{n}^{k}\left|f^{\left(q_{n}\right)}(x)\right| \leq K\|f\|_{k+\mathrm{BV}}
$$


for any $f \in C_{0}^{k+\mathrm{BV}}$ and $n \in \mathbb{N}$. Moreover, if $f \in C_{0}^{k+\mathrm{AC}}$, then the sequence $\left\{q_{n}^{k} f^{\left(q_{n}\right)}\right\}_{n \in \mathbb{N}}$ uniformly converges to zero.

Proof. Notice that Theorem 2.1 implies the first part of the corollary. Since for every $f \in C_{0}^{k+\mathrm{AC}}$ there exists a sequence $\left\{P_{m}\right\}_{m \in \mathbb{N}}$ of trigonometric polynomials with zero integral such that

$$
\lim _{m \rightarrow \infty}\left\|P_{m}-f\right\|_{k+\mathrm{BV}}=0,
$$

it suffices to show that for every trigonometric polynomial $f$ with zero integral the sequence $\left\{q_{n}^{k} f^{\left(q_{n}\right)}\right\}_{n \in \mathbb{N}}$ uniformly converges to zero. Let

$$
f(x)=\sum_{m=-M}^{M} a_{m} e^{2 \pi i m x}
$$

where $a_{0}=0$. Then

$$
\begin{aligned}
\left|q_{n}^{k} f^{\left(q_{n}\right)}(x)\right| & =\left|q_{n}^{k} \sum_{m=-M}^{M} a_{m} \frac{e^{2 \pi i m q_{n} \alpha}-1}{e^{2 \pi i m \alpha}-1} e^{2 \pi i m x}\right| \\
& \leq 2 q_{n}^{k} \sum_{m=-M}^{M}\left|a_{m}\right| \frac{m\left\|q_{n} \alpha\right\|}{\|m \alpha\|}=q_{n}^{k}\left\|q_{n} \alpha\right\| \sum_{m=-M}^{M} \frac{2\left|a_{m}\right| m}{\|m \alpha\|} .
\end{aligned}
$$

It follows that $q_{n}^{k} f^{\left(q_{n}\right)}$ uniformly converges to zero, which completes the proof.

3. Ergodicity of differentiable cocycles. We need auxiliary lemmas.

LemMA 3.1. Let $0=\beta_{0}<\beta_{1}<\ldots<\beta_{d}<\beta_{d+1}=1$ and let $a_{1}, \ldots, a_{d+1}$ be real numbers with zero sum. Consider a function $h: \mathbb{T} \rightarrow \mathbb{R}$ with zero integral given by

$$
h=h(0)+\sum_{i=1}^{d+1} a_{i} \mathbf{1}_{\left[\beta_{i}, 1\right)} .
$$

Then $h(0)=\sum_{i=1}^{d+1} a_{i} \beta_{i}$ and

$$
h^{(q)}=h^{(q)}(0)+\sum_{s=0}^{q-1} \sum_{i=1}^{d+1} a_{i} \mathbf{1}_{\left[\beta_{i}-s \alpha, 1\right)}
$$

for any natural $q$, where $T: \mathbb{T} \rightarrow \mathbb{T}$ is the rotation through $\alpha$.

Pro of. Since $\int_{\mathbb{T}} h d \lambda=0$ and $a_{1}+\ldots+a_{d+1}=0$, we have

$$
0=h(0)+\sum_{i=1}^{d+1} a_{i}\left(1-\beta_{i}\right)=h(0)-\sum_{i=1}^{d+1} a_{i} \beta_{i} .
$$


For all $a, b, x \in \mathbb{T}$, we have

$$
\mathbf{1}_{[b, 1)}(x+a)-\mathbf{1}_{[b, 1)}(a)=\mathbf{1}_{[b-a, 1)}(x)-\mathbf{1}_{[1-a, 1)}(x) .
$$

It follows that

$$
\begin{aligned}
h(x+a)-h(a) & =\sum_{i=1}^{d+1} a_{i}\left(\mathbf{1}_{\left[\beta_{i}, 1\right)}(x+a)-\mathbf{1}_{\left[\beta_{i}, 1\right)}(x)\right) \\
& =\sum_{i=1}^{d+1} a_{i}\left(\mathbf{1}_{\left[\beta_{i}-a, 1\right)}(x)-\mathbf{1}_{[1-a, 1)}(x)\right)=\sum_{i=1}^{d+1} a_{i} \mathbf{1}_{\left[\beta_{i}-a, 1\right)}(x) .
\end{aligned}
$$

Therefore

$$
h^{(q)}=h^{(q)}(0)+\sum_{s=0}^{q-1} \sum_{i=1}^{d+1} a_{i} \mathbf{1}_{\left[\beta_{i}-s \alpha, 1\right)}
$$

for any natural $q$.

Lemma 3.2. Let $I \subset \mathbb{R}$ be an interval and $k$ be a natural number. If $P$ is a real polynomial of the form $P(x)=c_{k} x^{k}+\ldots+c_{0}, c_{k} \neq 0$, then there exists a closed subinterval $J \subset I$ with $|J| \geq|I| / 4^{k}$ such that

$$
x \in J \Rightarrow|P(x)| \geq k !\left|c_{k}\right|(|I| / 4)^{k} .
$$

Proof. Let $f: \mathbb{R} \rightarrow \mathbb{R}$ be a differentiable function with continuous derivative. Suppose that there exists a closed interval $I \subset \mathbb{R}$ such that $|D f(x)| \geq a>0$ for any $x \in I$. We first show that there exists an interval $J \subset I$ with $|J| \geq|I| / 4$ and $|f(x)| \geq a|I| / 4$ for any $x \in J$. Without loss of generality we can assume that $D f(x) \geq a>0$ for any $x \in$ $I$. Suppose that for every interval $J \subset I$ with $|J| \geq|I| / 4$ there exists $x \in J$ such that $|f(x)|<a|I| / 4$. Since $f$ increases on $I$, we can find $x, y \in I$ such that $x-y \geq|I| / 2$ and $|f(x)|,|f(y)|<a|I| / 4$. It follows that

$$
a|I| / 2 \leq a|x-y| \leq|f(x)-f(y)|<a|I| / 2,
$$

a contradiction. Applying the above fact to derivatives of $P$ we obtain our assertion.

Let $f \in C_{0}^{k+\mathrm{PAC}}$ be such that $S\left(D^{k} f\right)=0$. Let $\alpha \in S_{k}^{0}$ and let $0=\beta_{0}<$ $\beta_{1}<\ldots<\beta_{d}<1$ be all the discontinuities of $D^{k} f$. Suppose that there exists a sequence $\left\{q_{n}\right\}_{n \in \mathbb{N}}$ of denominators of $\alpha$ such that

$$
\lim _{n \rightarrow \infty} q_{n}^{k+1}\left\|q_{n} \alpha\right\|=0 \quad \text { and } \quad \lim _{n \rightarrow \infty}\left\{q_{n} \beta_{i}\right\}=\gamma_{i},
$$

where $\gamma_{i} \neq \gamma_{j}$ for $i \neq j, i, j=0, \ldots, d$. It is clear that the function $f$ can 
be represented as $f=g+h$, where $g \in C_{0}^{k+\mathrm{AC}}, h \in C_{0}^{k+\mathrm{PAC}}$ and $D^{k} h$ is constant on each interval $\left(\beta_{i}, \beta_{i+1}\right)$. Then

$$
D^{k} h_{+}\left(\beta_{i}\right)-D^{k} h_{-}\left(\beta_{i}\right)=D^{k} f_{+}\left(\beta_{i}\right)-D^{k} f_{-}\left(\beta_{i}\right)=a_{i} \neq 0
$$

for $i=0, \ldots, d$ and

$$
D^{k} h_{+}=D^{k} h_{+}(0)+\sum_{i=1}^{d+1} a_{i} \mathbf{1}_{\left[\beta_{i}, 1\right)}
$$

with $D^{k} h_{+}(0)=\sum_{i=1}^{d+1} a_{i} \beta_{i}$. By Lemma 3.1,

$$
D^{k} h_{+}^{(q)}=D^{k} h_{+}^{(q)}(0)+\sum_{s=0}^{q-1} \sum_{i=1}^{d+1} a_{i} \mathbf{1}_{\left[\beta_{i}-s \alpha, 1\right)}
$$

for any natural $q$. Let $\sigma$ be a permutation of the set $\{0,1, \ldots, d\}$ such that

$$
0=\gamma_{\sigma(0)}<\gamma_{\sigma(1)}<\ldots<\gamma_{\sigma(d)}<\gamma_{\sigma(d+1)}=1,
$$

where $\sigma(0)=\sigma(d+1)$. For given $1 \leq i \leq d+1$ and $0 \leq j<q_{n}$, let $t_{i}^{(j)}$ be the unique integer satisfying $0 \leq t_{i}^{(j)}<q_{n}$ and

$$
t_{i}^{(j)} p_{n}+j=\left[q_{n} \beta_{i}\right] \bmod q_{n},
$$

where $\left\{p_{n} / q_{n}\right\}_{n \in \mathbb{N}}$ is the sequence of convergents of $\alpha$. Then

$$
\begin{aligned}
\beta_{i}-t_{i}^{(j)} \alpha & =\frac{\left[q_{n} \beta_{i}\right]}{q_{n}}+\frac{\left\{q_{n} \beta_{i}\right\}}{q_{n}}-t_{i}^{(j)} \frac{p_{n}}{q_{n}}-t_{i}^{(j)} \frac{\delta_{n}}{q_{n}} \\
& =\frac{j}{q_{n}}+\frac{1}{q_{n}}\left(\left\{q_{n} \beta_{i}\right\}-t_{i}^{(j)} \delta_{n}\right) \bmod 1,
\end{aligned}
$$

where $\left|\delta_{n}\right|=\left\|q_{n} \alpha\right\|$. It follows that

$$
\beta_{\sigma(0)}-t_{\sigma(0)}^{(j)} \alpha \widetilde{<} \beta_{\sigma(1)}-t_{\sigma(1)}^{(j)} \alpha \widetilde{<} \ldots \widetilde{<} \beta_{\sigma(d)}-t_{\sigma(d)}^{(j)} \alpha \widetilde{<} \beta_{\sigma(0)}-t_{\sigma(0)}^{(j+1)} \alpha
$$

for $j=0, \ldots, q_{n}-1$. Let $0 \leq j \leq q_{n}-1$ and $0 \leq i \leq d$. Set

$$
I_{i}^{(j)}= \begin{cases}\left(\beta_{\sigma(i)}-t_{\sigma(i)}^{(j)} \alpha, \beta_{\sigma(i+1)}-t_{\sigma(i+1)}^{(j)} \alpha\right) & \text { if } 0 \leq i<d, \\ \left(\beta_{\sigma(d)}-t_{\sigma(d)}^{(j)} \alpha, \beta_{\sigma(0)}-t_{\sigma(0)}^{(j+1)} \alpha\right) & \text { if } i=d .\end{cases}
$$

Lemma 3.3. If $x \in I_{i}^{(j)}$, then

$$
D^{k} h^{\left(q_{n}\right)}(x)=\sum_{m=1}^{d} a_{m}\left\{q_{n} \beta_{m}\right\}+\sum_{m=0}^{i} a_{m}
$$


Proof. Let $x \in I_{i}^{(j)}$. From (4), we have

$$
\begin{aligned}
D^{k} h^{\left(q_{n}\right)}(x) & =D^{k} h_{+}^{\left(q_{n}\right)}(0)+\sum_{l=0}^{q_{n}-1} \sum_{m=1}^{d+1} a_{m} \mathbf{1}_{\left[\beta_{\sigma(m)}-t_{\sigma(m)}^{(l)} \alpha, 1\right)}(x) \\
& =D^{k} h_{+}^{\left(q_{n}\right)}(0)+\sum_{l=0}^{j-1} \sum_{m=1}^{d+1} a_{m}+\sum_{m=1}^{d+1} a_{m} \mathbf{1}_{\left[\beta_{\sigma(m)}-t_{\sigma(m)}^{(j)} \alpha, 1\right)}(x) \\
& =D^{k} h_{+}^{\left(q_{n}\right)}(0)+\sum_{m=1}^{i} a_{m} .
\end{aligned}
$$

Moreover

$$
\begin{aligned}
D^{k} h_{+}^{\left(q_{n}\right)}(0) & =\sum_{j=0}^{q_{n}-1} D^{k} h_{+}(j \alpha)=\sum_{j=0}^{q_{n}-1}\left(D^{k} h_{+}(0)+\sum_{i=1}^{d} a_{i} \mathbf{1}_{\left[\beta_{i}, 1\right)}(j \alpha)\right) \\
& =q_{n} D^{k} h_{+}(0)+\sum_{i=1}^{d} a_{i} \sum_{j=0}^{q_{n}-1} \mathbf{1}_{\left[\beta_{i}, 1\right)}(j \alpha) .
\end{aligned}
$$

On the other hand,

$$
\begin{aligned}
& \sum_{j=0}^{q_{n}-1} \mathbf{1}_{\left[\beta_{i}, 1\right)}(j \alpha) \\
& \quad=\operatorname{card}\left\{0 \leq j<q_{n}:\{j \alpha\}>\beta_{i}\right\} \\
& \quad=\operatorname{card}\left\{0 \leq j<q_{n}:\left\{j p_{n} / q_{n}\right\}+j \delta_{n} / q_{n}>\left[q_{n} \beta_{i}\right] / q_{n}+\left\{q_{n} \beta_{i}\right\} / q_{n}\right\} \\
& \quad=\operatorname{card}\left\{0 \leq j<q_{n}:\left\{j p_{n} / q_{n}\right\}>\left[q_{n} \beta_{i}\right] / q_{n}\right\} \\
& \quad=q_{n}-\left[q_{n} \beta_{i}\right]-1 .
\end{aligned}
$$

Therefore

$$
\begin{aligned}
D^{k} h_{+}^{\left(q_{n}\right)}(0) & =q_{n} \sum_{i=1}^{d+1} a_{i} \beta_{i}+\sum_{i=1}^{d} a_{i}\left(q_{n}-\left[q_{n} \beta_{i}\right]-1\right) \\
& =q_{n} \sum_{i=1}^{d} a_{i} \beta_{i}+\sum_{i=1}^{d} a_{i}\left(\left\{q_{n} \beta_{i}\right\}-q_{n} \beta_{i}\right)+a_{0} \\
& =\sum_{i=1}^{d} a_{i}\left\{q_{n} \beta_{i}\right\}+a_{0}
\end{aligned}
$$

and consequently

$$
D^{k} h^{\left(q_{n}\right)}(x)=\sum_{m=1}^{d} a_{m}\left\{q_{n} \beta_{m}\right\}+\sum_{m=0}^{i} a_{m} .
$$


Let $0 \leq j \leq q_{n}-1$ and $0 \leq i \leq d$. Let $\widehat{I}_{i}^{(j)}$ denote the interval

$$
\left(\beta_{\sigma(i)}-t_{\sigma(i)}^{(j)} \alpha+q_{n}^{k}\left\|q_{n} \alpha\right\|, \beta_{\sigma(i+1)}-t_{\sigma(i+1)}^{(j)} \alpha-q_{n}^{k}\left\|q_{n} \alpha\right\|\right)
$$

if $0 \leq i<d$, and the interval

$$
\left(\beta_{\sigma(d)}-t_{\sigma(d)}^{(j)} \alpha+q_{n}^{k}\left\|q_{n} \alpha\right\|, \beta_{\sigma(0)}-t_{\sigma(0)}^{(j+1)} \alpha-q_{n}^{k}\left\|q_{n} \alpha\right\|\right)
$$

if $i=d$. Since $q_{n}^{k+1}\left\|q_{n} \alpha\right\| \rightarrow 0$ as $n \rightarrow \infty$, we have

$$
\begin{aligned}
\left|\widehat{I}_{i}^{(j)}\right| & =\frac{1}{q_{n}}\left|\left\{q_{n} \beta_{\sigma(i+1)}\right\}-\left\{q_{n} \beta_{\sigma(i)}\right\}-\delta_{n}\left(t_{\sigma(i+1)}^{(j)}-t_{\sigma(i)}^{(j)}\right)-2 q_{n}^{k+1}\left\|q_{n} \alpha\right\|\right| \\
& \geq \frac{\gamma_{\sigma(i+1)}-\gamma_{\sigma(i)}}{2 q_{n}}
\end{aligned}
$$

for all $n$ large enough.

Corollary 3.1. If $x \in \widehat{I}_{i}^{(j)}$, then

$$
D^{k} h^{\left(q_{n}^{k+1}\right)}(x)=q_{n}^{k}\left(\sum_{m=1}^{d} a_{m}\left\{q_{n} \beta_{m}\right\}+\sum_{m=0}^{i} a_{m}\right) .
$$

Proof. For every $x \in \mathbb{T}$, we have

$$
\begin{aligned}
D^{k} h^{\left(q_{n}^{k+1}\right)}(x)= & D^{k} h^{\left(q_{n}\right)}(x) \\
& +D^{k} h^{\left(q_{n}\right)}\left(x+q_{n} \alpha\right)+\ldots+D^{k} h^{\left(q_{n}\right)}\left(x+\left(q_{n}^{k}-1\right) q_{n} \alpha\right) .
\end{aligned}
$$

If $x \in \widehat{I}_{i}^{(j)}$, then $x+l q_{n} \alpha \in I_{i}^{(j)}$ for $l=0,1, \ldots, q_{n}^{k}-1$. It follows that

$$
D^{k} h^{\left(q_{n}^{k+1}\right)}(x)=q_{n}^{k}\left(\sum_{m=1}^{d} a_{m}\left\{q_{n} \beta_{m}\right\}+\sum_{m=0}^{i} a_{m}\right) .
$$

Corollary 3.2. There exists a collection $\left\{J_{j}\right\}_{j=0}^{q_{n}-1}$ of pairwise disjoint closed intervals and there exist constants $0<C<1, M>0$ such that

$$
\left|J_{j}\right| \geq \frac{C}{q_{n}} \text { and } x \in J_{j} \Rightarrow\left|D h^{\left(q_{n}^{k+1}\right)}(x)\right| \geq M q_{n}
$$

for $j=0, \ldots, q_{n}-1$.

Proof. Fix

$$
c_{i}=\sum_{m=1}^{d} a_{m} \gamma_{m}+\sum_{m=0}^{i} a_{m} .
$$

At least one of the numbers $c_{i}$ is not zero. Indeed, if we suppose that $c_{i}=0$ for $i=0, \ldots, d$, then $a_{i}=c_{i}-c_{i-1}=0$ for $i=0, \ldots, d$, which is impossible. Take $i_{0}$ such that $c_{i_{0}} \neq 0$. Set

$$
b^{(n)}=\sum_{m=1}^{d} a_{m}\left\{q_{n} \beta_{m}\right\}+\sum_{m=0}^{i_{0}} a_{m} .
$$


Since $D^{k} h^{\left(q_{n}^{k+1}\right)}=q_{n}^{k} b^{(n)}$ on $\widehat{I}_{i_{0}}^{(j)}$, we have

$$
D h^{\left(q_{n}^{k+1}\right)}(x)=q_{n}^{k} b^{(n)} x^{k-1}+P_{j}(x)
$$

on $\widehat{I}_{i_{0}}^{(j)}$, where $P_{j}$ is a polynomial with $\operatorname{deg}\left(P_{j}\right)<k-1\left(j=0, \ldots, q_{n}-1\right)$. By Lemma 3.2, there exist closed subintervals $J_{j} \subset \widehat{I}_{i_{0}}^{(j)}$ such that

$$
\left|J_{j}\right| \geq \frac{1}{4^{k-1}}\left|\widehat{I}_{i_{0}}^{(j)}\right| \geq \frac{\gamma_{\sigma\left(i_{0}+1\right)}-\gamma_{\sigma\left(i_{0}\right)}}{4^{k} q_{n}}
$$

and if $x \in J_{j}$, then

$$
\begin{aligned}
\left.\mid D h^{\left(q_{n}^{k+1}\right)}(x)\right) \mid & \geq q_{n}^{k}\left|b^{(n)}\right|\left(\frac{\left|\widehat{I}_{i_{0}}^{(j)}\right|}{4}\right)^{k-1} \geq \frac{1}{2} q_{n}^{k}\left|c_{i_{0}}\right|\left(\frac{\gamma_{\sigma\left(i_{0}+1\right)}-\gamma_{\sigma\left(i_{0}\right)}}{4^{k} q_{n}}\right)^{k-1} \\
& \geq q_{n} \frac{\left|c_{i_{0}}\right|\left(\gamma_{\sigma\left(i_{0}+1\right)}-\gamma_{\sigma\left(i_{0}+1\right)}\right)^{k-1}}{4^{k^{2}}}
\end{aligned}
$$

for $j=0, \ldots, q_{n}-1$. It follows that we can set

$$
C=\frac{\gamma_{\sigma\left(i_{0}+1\right)}-\gamma_{\sigma\left(i_{0}\right)}}{4^{k}} \quad \text { and } \quad M=\frac{\left|c_{i_{0}}\right|\left(\gamma_{\sigma\left(i_{0}+1\right)}-\gamma_{\sigma\left(i_{0}\right)}\right)^{k-1}}{4^{k^{2}}} .
$$

Proof of Theorem 1.1. Notice that $\left\{q_{n}^{k+1}\right\}_{n \in \mathbb{N}}$ is a rigid time for the rotation $T x=x+\alpha$. By Corollary 2.1, the sequence $\left\{\left\|(f+v)^{\left(q_{n}^{k+1}\right)}\right\|_{\infty}\right\}_{n \in \mathbb{N}}$ is bounded, because $\left\|(f+v)^{\left(q_{n}^{k+1}\right)}\right\|_{\infty} \leq q_{n}^{k}\left\|(f+v)^{\left(q_{n}\right)}\right\|_{\infty}$ and $f+v \in C_{0}^{k+\mathrm{BV}}$. By Proposition 1, it suffices to find $\varepsilon>0$ such that $\operatorname{Var}\left(D^{k} v\right)<\varepsilon$ implies

$$
\limsup _{n \rightarrow \infty}\left|\int_{\mathbb{T}} e^{2 \pi i l(f+v)^{\left(q_{n}^{k+1}\right)}(x)} d x\right| \leq c<1
$$

for all $l$ large enough.

Represent $f$ as the sum of functions $g \in C_{0}^{k+\mathrm{AC}}$ and $h \in C_{0}^{k+\mathrm{PAC}}$, where $D^{k} h$ is constant on intervals $\left(\beta_{i}, \beta_{i+1}\right)$. Since $\left\|g^{\left(q_{n}^{k+1}\right)}\right\|_{\infty} \leq q_{n}^{k}\left\|g^{\left(q_{n}\right)}\right\|_{\infty}$, the sequence $\left\{g^{\left(q_{n}^{k+1}\right)}\right\}_{n \in \mathbb{N}}$ uniformly converges to zero, by Corollary 2.1. Therefore

$$
\lim _{n \rightarrow \infty}\left|\int_{\mathbb{T}} e^{2 \pi i l(f+v)^{\left(q_{n}^{k+1}\right)}(x)} d x-\int_{\mathbb{T}} e^{2 \pi i l(h+v)^{\left(q_{n}^{k+1}\right)}(x)} d x\right|=0 .
$$

It follows that it suffices to compute

$$
\limsup _{n \rightarrow \infty}\left|\int_{\mathbb{T}} e^{2 \pi i l(h+v)^{\left(q_{n}^{k+1}\right)}(x)} d x\right| .
$$

By Corollary 3.2, there exists a collection $\left\{J_{j}: j=0, \ldots, q_{n}-1\right\}$ of pairwise disjoint closed intervals and there exist $0<C<1, M>0$ such that

$$
\left|J_{j}\right| \geq \frac{C}{q_{n}} \text { and } x \in J_{j} \Rightarrow\left|D h^{\left(q_{n}^{k+1}\right)}(x)\right| \geq M q_{n}
$$


for $j=0, \ldots, q_{n}-1$. Let $J_{j}=\left[a_{j}, b_{j}\right]$ for $j=0, \ldots, q_{n}-1$. Applying integration by parts we get

$$
\begin{aligned}
&\left|\int_{\mathbb{T}} e^{2 \pi i l(h+v)^{\left(q_{n}^{k+1}\right)}(x)} d x\right| \\
& \leq 1-\sum_{j=0}^{q_{n}-1}\left|J_{j}\right|+\left|\sum_{j=0}^{q_{n}-1} \int_{a_{j}}^{b_{j}} e^{2 \pi i l(h+v)^{\left(q_{n}^{k+1}\right)}(x)} d x\right| \\
& \leq 1-C+\left|\sum_{j=0}^{q_{n}-1} \int_{a_{j}}^{b_{j}} \frac{e^{2 \pi i l v^{\left(q_{n}^{k+1}\right)}(x)}}{2 \pi i l D h^{\left(q_{n}^{k+1}\right)}(x)} d e^{2 \pi i l h^{\left(q_{n}^{k+1}\right)}(x)}\right| \\
&=1-C \\
&+\mid \sum_{j=0}^{q_{n}-1}\left(\frac{e^{2 \pi i l(h+v)^{\left(q_{n}^{k+1}\right)}\left(b_{j}\right)}}{2 \pi i l D h^{\left(q_{n}^{k+1}\right)}\left(b_{j}\right)}-\frac{e^{2 \pi i l(h+v)^{\left(q_{n}^{k+1}\right)}\left(a_{j}\right)}}{2 \pi i l D h^{\left(q_{n}^{k+1}\right)}\left(a_{j}\right)}\right. \\
&\left.-\int_{a_{j}}^{b_{j}} e^{2 \pi i l h^{\left(q_{n}^{k+1}\right)}(x)} d \frac{e^{2 \pi i l v^{\left(q_{n}^{k+1}\right)}(x)}}{2 \pi i l D h^{\left(q_{n}^{k+1}\right)}(x)}\right) \mid .
\end{aligned}
$$

Since $\left|D h^{\left(q_{n}^{k+1}\right)}(x)\right| \geq M q_{n}$ for every $x \in J_{j}$, we obtain

$$
\left|\sum_{j=0}^{q_{n}-1}\left(\frac{e^{2 \pi i l(h+v)^{\left(q_{n}^{k+1}\right)}\left(b_{j}\right)}}{2 \pi i l D h^{\left(q_{n}^{k+1}\right)}\left(b_{j}\right)}-\frac{e^{2 \pi i l(h+v)^{\left(q_{n}^{k+1}\right)}\left(a_{j}\right)}}{2 \pi i l D h^{\left(q_{n}^{k+1}\right)}\left(a_{j}\right)}\right)\right| \leq \frac{1}{l M \pi}
$$

and

$$
\begin{aligned}
\left|\int_{a_{j}}^{b_{j}} e^{2 \pi i l h^{\left(q_{n}^{k+1}\right)}(x)} d \frac{e^{2 \pi i l v^{\left(q_{n}^{k+1}\right)}(x)}}{D h^{\left(q_{n}^{k+1}\right)}(x)}\right| & \leq \operatorname{Var}_{a_{j}}^{b_{j}}\left(\frac{e^{2 \pi i l v^{\left(q_{n}^{k+1}\right)}}}{D h^{\left(q_{n}^{k+1}\right)}}\right) \\
& \leq \frac{2 \pi l \operatorname{Var}_{a_{j}}^{b_{j}}\left(v^{\left(q_{n}^{k+1}\right)}\right)}{\inf _{\left(a_{j}, b_{j}\right)}\left|D h^{\left(q_{n}^{k+1}\right)}\right|}+\operatorname{Var}_{a_{j}}^{b_{j}}\left(\frac{1}{D h^{\left(q_{n}^{k+1}\right)}}\right) \\
& \leq \frac{2 \pi l}{M q_{n}} \int_{a_{j}}^{b_{j}}\left|D v^{\left(q_{n}^{k+1}\right)}\right| d \lambda+\frac{\operatorname{Var}_{a_{j}}^{b_{j}}\left(D h^{\left(q_{n}^{k+1}\right)}\right)}{M^{2} q_{n}^{2}}
\end{aligned}
$$

for $j=0, \ldots, q_{n}-1$. It follows that

$$
\begin{aligned}
\left|\int_{\mathbb{T}} e^{2 \pi i l(h+v)^{\left(q_{n}^{k+1}\right)}(x)} d x\right| \leq & -C+\frac{1}{l M \pi} \\
& +\frac{1}{M q_{n}} \int_{\mathbb{T}}\left|D v^{\left(q_{n}^{k+1}\right)}\right| d \lambda+\frac{\operatorname{Var}\left(D h^{\left(q_{n}^{k+1}\right)}\right)}{2 \pi l M^{2} q_{n}^{2}} .
\end{aligned}
$$


By Corollary 2.1, we have

$$
\int_{\mathbb{T}}\left|D v^{\left(q_{n}^{k+1}\right)}\right| d \lambda \leq q_{n}^{k} \int_{\mathbb{T}}\left|D v^{\left(q_{n}\right)}\right| d \lambda \leq K q_{n}\|v\|_{k+\mathrm{BV}} .
$$

Moreover,

$$
\operatorname{Var}\left(D h^{\left(q_{n}^{k+1}\right)}\right) \leq K q_{n}^{2}\|h\|_{k+\mathrm{BV}} .
$$

Indeed, for $k=1$, we have

$$
\operatorname{Var}\left(D h^{\left(q_{n}^{k+1}\right)}\right) \leq q_{n}^{2} \operatorname{Var}(D h)
$$

and

$$
\operatorname{Var}\left(D h^{\left(q_{n}^{k+1}\right)}\right)=\int_{\mathbb{T}}\left|D^{2} h^{\left(q_{n}^{k+1}\right)}\right| d \lambda \leq q_{n}^{k} \int_{\mathbb{T}}\left|D^{2} h^{\left(q_{n}\right)}\right| d \lambda \leq K q_{n}^{2} \operatorname{Var}\left(D^{k} h\right)
$$

for $k>1$, by Corollary 2.1. It follows that

$\limsup _{n \rightarrow \infty}\left|\int_{\mathbb{T}} e^{2 \pi i l(h+v)^{\left(q_{n}^{k+1}\right)}(x)} d x\right| \leq 1-C+\frac{1}{l M \pi}+\frac{K}{M}\|v\|_{k+\mathrm{BV}}+\frac{K}{l M^{2}}\|h\|_{k+\mathrm{BV}}$.

Let $v \in C_{0}^{k+\mathrm{BV}}$. Suppose that $\|v\|_{k+\mathrm{BV}}<M C / K$. Then

$$
\limsup _{n \rightarrow \infty}\left|\int_{\mathbb{T}} e^{2 \pi i l(h+v)^{\left(q_{n}^{k+1}\right)}(x)} d x\right| \leq 1-\frac{1}{2}\left(C-\frac{K}{M}\|v\|_{k+\mathrm{BV}}\right)<1
$$

for all $l$ large enough, which completes the proof.

\section{References}

[1] I. P. Cornfeld, S. V. Fomin and Ya. G. Sinai, Ergodic Theory, Springer, Berlin, 1982.

[2] H. Furstenberg, Strict ergodicity and transformations on the torus, Amer. J. Math. 83 (1961), 573-601.

[3] P. Gabriel, M. Lemańczyk et P. Liardet, Ensemble d'invariants pour les produits croisés de Anzai, Mém. Soc. Math. France 47 (1991).

[4] P. Hellekalek and G. Larcher, On the ergodicity of a class of skew products, Israel J. Math. 54 (1986), 301-306.

[5] M. R. Herman, Sur la conjugaison différentiable des difféomorphismes du cercle à des rotations, Publ. Mat. IHES 49 (1979), 5-234.

[6] M. Lemańczyk, F. Parreau and D. Volný, Ergodic properties of real cocycles and pseudo-homogeneous Banach spaces, Trans. Amer. Math. Soc. 348 (1996), 49194938.

[7] W. Parry, Topics in Ergodic Theory, Cambridge Univ. Press, Cambridge, 1981.

[8] D. Pask, Skew products over the irrational rotation, Israel J. Math. 69 (1990), 65-74.

[9] -, Ergodicity of certain cylinder flows, ibid. 76 (1991), 129-152. 
[10] K. Schmidt, Cocycles of Ergodic Transformation Groups, Macmillan Lectures in Math. 1, Delhi, 1977.

Faculty of Mathematics and Computer Science

Nicholas Copernicus University

Chopina 12/18

87-100 Toruń, Poland

E-mail: fraczek@mat.uni.torun.pl

Received 16 November 1998 